\title{
SOME ADDITIONAL REMARKS ON THE CUMULANT EXPANSION FOR LINEAR STOCHASTIC DIFFERENTIAL EQUATIONS
}

\author{
J.B.T.M. ROERDINK*
}

Institute for Theoretical Physics, Princetonplein 5, P.O. Box 80.006, 3508 TA Utrecht, The Netherlands

Received 15 July 1983

\begin{abstract}
We summarize our previous results on cumulant expansions for linear stochastic differential equations with correlated multipliclative and additive noise. The application of the general formulas to equations with statistically independent multiplicative and additive noise is reconsidered in detail, because our earlier results for this case are only valid in special situations. Moreover, a second partially time ordered cumulant is introduced, which is appropriate for the calculation of multi-time averages of the solution process and which differs slightly from the one to be used for single-time averages. By the two supplements just mentioned we complete our earlier investigations on the subject.
\end{abstract}

\section{Introduction}

In two earlier papers, henceforth referred to as $\mathrm{I}^{1}$ ) and $\mathrm{II}^{2}$ ), we discussed the cumulant expansion for linear inhomogeneous stochastic differential equations with correlated multiplicative and additive noise. Results for single- ${ }^{1}$ ) and multi-time ${ }^{2}$ ) averages were obtained. To deal with inhomogeneous equations a partially time-ordered cumulant (p-cumulant) was introduced in I. This pcumulant has some peculiar properties which have not completely been recognized before. As a consequence our discussion in section 6.1 of $\mathrm{I}$ is not fully correct (except in special cases; the problem of an harmonic oscillator with a deltacorrelated stochastic frequency, which we discussed in subsection 6.2 of I, constitutes such a case; see subsection 2.2). The general results of section 4 of I remain correct, however.

Moreover, in the case of multi-time averages the partially time-ordered cumulant has to be interpreted slightly differently from the p-cumulant in the case of single-time averages. Therefore we introduce a new partially time-ordered cumulant, abbreviated as $\overline{\mathrm{p}}$-cumulant, thus providing the correct interpretation of the cumulants as used in II.

We would like to stress here that all our previous results up to second order in the multiplicative and/or additive noise are unaffected by the two modifications just mentioned. We treat the cases of single- and multi-time averages in two

* Future address: Department of Chemistry, UCSD, La Jolla, CA 92093, USA.

0378-4371/84/\$03.00 (C) Elsevier Science Publishers B.V.

(North-Holland Physics Publishing Division) 
different sections 2 and 3, respectively. For the sake of clarity, each section is subdivided in a subsection which summarizes the (corrected) results, and a subsequent subsection which contains a discussion of the modifications in these results as compared to our earlier treatments I and II. New derivations, if necessary, are delegated to a number of appendices.

\section{The cumulant expansion for single-time averages}

In this section we discuss the inhomogeneous equation

$$
\begin{aligned}
& \dot{u}(t)=\left\{A_{0}+\alpha A_{1}(t)\right\} u(t)+f(t), \\
& u\left(t_{0}\right)=u_{0},
\end{aligned}
$$

where $u$ is a vector, $A_{0}$ a deterministic and $A_{1}(t)$ a stochastic matrix, and $f(t)$ a stochastic vector. All quantities $A_{1}, f$ and $u_{0}$ are not only random, but also mutually correlated. The central assumption which underlies the cumulant expansion is that the autocorrelation time of $A_{1}(t)$ and the cross correlation times of $A_{1}(t)$ with $f(t)$ and with $u_{0}$ are all finite with maximum $\tau_{\mathrm{c}}$. The expansion then proceeds in powers of $\alpha \tau_{c}$. For the validity of the expansion one has to require that the condition $\alpha \tau_{c} \ll 1$ is satisfied.

As usual we transform (2.1) to the interaction representation by defining

$$
\begin{aligned}
& v(t)=\mathrm{e}^{-\left(t-t_{0}\right) A_{0}} u(t), \\
& V(t)=\alpha \mathrm{e}^{-\left(t-t_{0}\right) A_{0}} A_{1}(t) \mathrm{e}^{\left(t-t_{0}\right) A_{0}}, \quad g(t)=\mathrm{e}^{-\left(t-t_{0}\right) A_{0}} f(t) .
\end{aligned}
$$

Then (2.1) transforms to

$$
\begin{aligned}
& \dot{v}(t)=V(t) v(t)+g(t), \\
& v\left(t_{0}\right)=u_{0},
\end{aligned}
$$

where $V(t)$ is now of order $\alpha$. In the rest of this paper we mainly discuss eq. (2.3). For results in the original representation we refer to I and II.

\subsection{Results and discussion}

\subsubsection{Results}

As shown in I the average of $v(t)$ obeys the equation

$$
\frac{\mathrm{d}}{\mathrm{d} t}\langle v(t)\rangle=K_{V}\left(t / t_{0}\right)\langle v(t)\rangle+F_{V}\left(t / t_{0}\right)+I_{V}\left(t / t_{0}\right),
$$


where

$$
\begin{aligned}
& K_{V}\left(t / t_{0}\right)=\left\langle V(t): Q_{V}\left(t / t_{0}\right):\right\rangle_{\mathrm{p}}, \\
& F_{V}\left(t / t_{0}\right)=\langle g(t)\rangle+\int_{t_{0}}^{t} \mathrm{~d} s\left\langle V(t): Q_{V}(t / s): g(s)\right\rangle_{\mathrm{p}}, \\
& I_{V}\left(t / t_{0}\right)=\left\langle V(t)\left(u_{0}-\left\langle u_{0}\right\rangle\right)\right\rangle+\int_{t_{0}}^{t} \mathrm{~d} s\left\langle V(t): Q_{V}(t / s):\left[V(s)\left(u_{0}-\left\langle u_{0}\right\rangle\right)\right]\right\rangle_{\mathrm{p}} .
\end{aligned}
$$

Here we use the short hand notation introduced in II. That is, for an arbitrary matrix $V(t)$ we define

$$
Q_{V}\left(t / t^{\prime}\right)=\grave{T}\left[\exp \int_{t^{\prime}}^{t} \mathrm{~d} s V(s)\right]\left(t \geqslant t^{\prime}\right)
$$

where $\overleftarrow{T}$ denotes time ordering (latest times to the left). The colons in (2.5) indicate that one should first expand the expressions between these colons in powers of $V$ and subsequently calculate the p-cumulant $\langle\ldots\rangle_{p}$ for each term. The definition of the p-cumulant is given in appendix $\mathbf{A}$.

The utility of eq. (2.4) rests upon the fact that $K_{V}$ and $F_{V}$ are independent of $t_{0}$, while $I_{V}$ vanishes, if $t-t_{0} \gg \tau_{\mathrm{c}}$.

Note that the results $(2.4)$ and (2.5) are exactly the same as in section 4 of $I$.

\subsubsection{Discussion}

In section 3.3 of I we asserted that $F_{V}$ in $(2.5 b)$ reduces to $\langle g(t)\rangle$ in the case that $V$ and $g$ in (2.3) are statistically independent, because joint cumulants of $V$ with $g$ were assumed to vanish. This however is not true in general. For this reason eq. (1.2) of $I$ is in general also incorrect (for the correct formula, see appendix B).

To see how this comes about, consider the cumulants of $V$ with $g$, assuming that $V$ and $g$ are statistically independent. The second order cumulant indeed vanishes,

$$
\left\langle V(t) g\left(t_{1}\right)\right\rangle_{\mathrm{p}}=\left\langle V(t) g\left(t_{1}\right)\right\rangle-\langle V(t)\rangle\left\langle g\left(t_{1}\right)\right\rangle=0,
$$

but the third order cumulant does not,

$$
\begin{aligned}
\left\langle V(t) V\left(t_{1}\right) g\left(t_{2}\right)\right\rangle_{\mathrm{p}} & =\left\langle V(t) V\left(t_{1}\right) g\left(t_{2}\right)\right\rangle_{\mathrm{t}}-\left\langle V(t) V\left(t_{2}\right)\right\rangle_{\mathrm{t}}\left\langle g\left(t_{1}\right)\right\rangle \\
& =-\left\langle V(t) V\left(t_{2}\right)\right\rangle_{\mathrm{t}}\left\langle g\left(t_{1}\right)\right\rangle .
\end{aligned}
$$

Here we used the decomposition of the p-cumulant in products of t-cumulants according to the rules in appendix $A$ of $I$. Note that any t-cumulant with a terminal 
vector $g$ and any number of matrices $V$ in front does vanish,

$$
\left\langle V(t) V\left(t_{1}\right) \ldots V\left(t_{m-1}\right) g\left(t_{m}\right)\right\rangle_{\mathrm{t}}=0 .
$$

For, in terms of the averaging operator $\mathscr{P} \ldots=\langle\ldots\rangle$, the t-cumulant equals

$$
\begin{aligned}
& \left\langle V(t) \ldots V\left(t_{m-1}\right) g\left(t_{m}\right)\right\rangle_{1} \equiv\left\langle V(t)(1-\mathscr{P}) \ldots V\left(t_{m-1}\right)(1-\mathscr{P}) g\left(t_{m}\right)\right\rangle \\
& \quad=\left\langle V(t)(1-\mathscr{P}) \ldots V\left(t_{m-1}\right) g\left(t_{m}\right)\right\rangle-\left\langle V(t)(1-\mathscr{P}) \ldots V\left(t_{m-1}\right)\right\rangle\left\langle g\left(t_{m}\right)\right\rangle=0,
\end{aligned}
$$

since $V$ and $g$ are statistically independent. Also any t-cumulant with an initial factor $A(t)$ independent of any number of subsequent quantities $B(\cdot)$ vanishes,

$$
\left\langle A(t) B\left(t_{1}\right) \ldots B\left(t_{m}\right)\right\rangle_{\mathrm{t}}=0,
$$

but in general (due to the noncommutativity of the quantities involved)

$$
\left\langle B(t) \ldots B\left(t_{k}\right) A\left(t_{k+1}\right) B\left(t_{k+2}\right) \ldots B\left(t_{m}\right)\right\rangle_{\mathrm{t}} \neq 0 .
$$

Although (2.10) also holds for p-cumulants (expressed in products of t-cumulants there is always a factor of the form (2.10) with $m \geqslant 1$ in front), the property (2.9) apparently does not hold for p-cumulants. However, if $\langle g(t)\rangle=0$, p-cumulants and t-cumulants with matrices $V$ and only one vector $g$ vanish, because one can first average over the fluctuations in $g$ which have zero average.

Hence, if $g$ is statistically independent of $V$ and $\langle g(t)\rangle=0, F_{V}\left(t / t_{0}\right)$ as given by (2.5b) vanishes identically. By the same argument, $I_{V}\left(t / t_{0}\right)=0$ in $(2.5 \mathrm{c})$ if $u_{0}$ is nonrandom or statistically independent of $V$ (because $\left\langle u_{0}-\left\langle u_{0}\right\rangle\right\rangle=0$ too).

Remark. The usefulness of the p-cumulants $\left\langle A_{0}(t) A_{1}\left(t_{1}\right) \ldots A_{m}\left(t_{m}\right)\right\rangle_{\mathrm{p}}$ (where $A_{m}$ is a matrix or vector) follows from the fact that they vanish if there is a gap between any two successive time-variables $t_{i}$ and $t_{i+1}$ which is large compared to $\tau_{\mathrm{c}}$. Here it is essential that the times are ordered, $t \geqslant t_{1} \geqslant \cdots \geqslant t_{m}$, so that for arbitrary $k$ and $l$ all quantities $A_{k}\left(t_{p}\right)$ are statistically independent of $A_{l}\left(t_{q}\right)$ if $t_{p} \geqslant t_{i}$ and $t_{q} \leqslant t_{i+1}$. This implies that the p-cumulant in (2.8) does vanish if $t-t_{1} \gg \tau_{c}$ or if $t_{1}-t_{2} \gg \tau_{\mathrm{c}}$. So for $\tau_{\mathrm{c}}$ one should really use the maximum of the autocorrelation time $\tau_{\mathrm{v}}$ of $V$ and the crosscorrelation time $\tau_{v g}$ of $V$ with $g$ ( $\tau_{v g}$ is zero if $V$ and $g$ are statistically independent, as in (2.8)). The cumulant in (2.8) vanishes if not only $A(t)$ and $A\left(t_{1}\right)$ are independent of $f\left(t_{2}\right)$, but also $A(t)$ is independent of $A\left(t_{2}\right)$; hence $t_{1}-t_{2} \gg \tau_{\mathrm{v}}=\tau_{\mathrm{c}}$ is required.

\subsection{Second moments}

In section 6.1 of I we studied the second moments of the solution to (2.1) by introducing the vector

$$
U(t)=\left(\begin{array}{l}
u(t) \\
u(t) \otimes u(t)
\end{array}\right),
$$


where $\otimes$ denotes a Kronecker product. This vector obeys the equation*

$$
\dot{U}(t)=\left\{\mathscr{A}_{0}+\mathscr{A}_{1}(t)\right\} U(t)+\mathscr{F}(t),
$$

where

$$
\mathscr{A}_{0}=\left(\begin{array}{c:c}
A_{0} & \emptyset \\
\hdashline \emptyset & \tilde{A}_{0}
\end{array}\right) ; \quad \mathscr{A}_{1}(t)=\left(\begin{array}{c:c}
\alpha A_{1}(t) & \emptyset \\
\hdashline \tilde{f}(t) & \alpha \tilde{A}_{1}(t)
\end{array}\right) ; \quad \mathscr{F}(t)=\left(\begin{array}{c}
f(t) \\
\emptyset
\end{array}\right) .
$$

Here we define for an arbitrary vector or matrix $C$,

$$
C^{\prime}=C \otimes \hat{1}, \quad C^{\prime \prime}=\hat{1} \otimes C, \quad \tilde{C}=C^{\prime}+C^{\prime \prime} .
$$

Here $\hat{1}$ denotes a unit matrix of the same dimension as $C$.

Since (2.12) is of the same form as (2.1), the general results of subsection 2.1.1 are immediately applicable. The result is that the average of $U(t)$ obeys

$$
\frac{\mathrm{d}}{\mathrm{d} t}\langle U(t)\rangle=\mathbb{K}\left(t / t_{0}\right)\langle U(t)\rangle+\mathbb{F}\left(t / t_{0}\right),
$$

where the contributions to $\mathbb{K}$ and $\mathbb{F}$ up to second order are given by eqs. (6.6), (6.8) and (6.9) of I. In general higher order cumulants involving $A_{1}$ and $f$ are nonzero, even if $A_{1}$ and $f$ are independent (see subsection 2.1.2). Therefore the formula (6.11) of $\mathrm{I}$ is in general incorrect. However, it is shown in appendix $\mathrm{B}$, that in the special case that $A_{1}$ and $f$ in (2.1) are statistically independent, and moreover $A_{1}$ has delta-correlated cumulants, i.e.

$$
\begin{aligned}
& \left\langle A_{1}\left(t_{1}\right)\right\rangle=D_{1}\left(t_{1}\right), \\
& \left\langle A_{1}\left(t_{1}\right) \ldots A_{1}\left(t_{m}\right)\right\rangle=D_{m}\left(t_{1}\right) \delta\left(t_{1}-t_{2}\right) \ldots \delta\left(t_{m-1}-t_{m}\right), \quad(m \geqslant 2),
\end{aligned}
$$

where $D_{m}(t)$ is a (time-dependent) matrix, the matrix $\mathbb{K}$ and the vector $\mathbb{F}$ in $(2.15)$ are given by the exact expressions

$$
\begin{aligned}
& \mathbb{K}\left(t / t_{0}\right)=\left(\begin{array}{c:c}
K\left(t / t_{0}\right) & \emptyset \\
\hdashline\langle\bar{f}(t)\rangle & \tilde{K}\left(t / t_{0}\right)
\end{array}\right), \\
& \mathbb{F}\left(t / t_{0}\right)=\left(\begin{array}{c}
\langle f(t)\rangle \\
\left.\int_{t_{0}} \mathrm{~d} s \mathrm{e}^{\left(t-t_{0}\right) \tilde{A}_{0}}\left\langle\left\langle\tilde{g}(t)\left\{\left\langle Q_{V}(t / s)\right\rangle g(s)\right\}\right\rangle\right\rangle\right\rangle
\end{array}\right) .
\end{aligned}
$$

The matrix $K$ is given by

$$
K\left(t / t_{0}\right)=A_{0}+\mathrm{e}^{\left(t-t_{0}\right) A_{0}} K_{V}\left(t / t_{0}\right) \mathrm{e}^{-\left(t-t_{0}\right) A_{0}},
$$

* With $\emptyset$ we indicate a null-matrix or null-vector. 
where $V$ and $K_{V}$ are defined in (2.2b) and (2.5a), respectively. $\tilde{K}$ is obtained from $K$ by the prescription (2.14) and $g$ is defined in (2.2b). Using the results of section 2 of I we can also write

$$
\left\langle Q_{V}(t / s)\right\rangle=\overleftarrow{T}\left[\exp \int_{s}^{t} \mathrm{~d} s^{\prime} K_{V}\left(s^{\prime} / s\right)\right]
$$

Remark. For the case of the harmonic oscillator problem studied in section 6.2 of $I$, the formulas (2.17) and (2.18) do apply, since the frequency fluctuations were assumed to be delta-correlated and independent of the additive fluctuations. Since we found by explicit calculation that for this model $K_{V}\left(s^{\prime} / s\right)=0$, i.e. $\left\langle Q_{V}(t / s)\right\rangle=1$, the formulas (2.17) and (2.18) reduce to those in (6.11) of I. This explains why application of the latter formula (although in general incorrect) nevertheless yielded the correct answer in section 6.2 of $\mathrm{I}$.

\section{The cumulant expansion for multi-time averages}

In this section we state and discuss the results of the cumulant expansion for multi-time averages.

\subsection{The homogeneous case}

\subsubsection{Results}

The correlation function $C_{v}(t, t+\tau) \equiv\langle v(t) \otimes v(t+\tau)\rangle$ of the solution of (2.3) with $g=0$ obeys the differential equation

$$
\frac{\partial}{\partial \tau} C_{v}(t, t+\tau)=M_{V}\left(t+\tau ; t / t_{0}\right) C_{v}(t, t+\tau),
$$

with

$$
M_{V}\left(t+\tau ; t / t_{0}\right)=\left\langle V^{\prime \prime}(t+\tau): Q_{V^{\prime}}(t+\tau / t) Q_{\nu}\left(t / t_{0}\right):\right\rangle_{\overline{\mathbf{p}}}
$$

Here the suffix $\bar{p}$ denotes a modified partially time-ordered cumulant, which differs from a p-cumulant only by the fact that matrices themselves are permuted, and not only their time variables. The precise definition of $p$ - and $\bar{p}$-cumulants is given in appendix $A$. If only one type of matrix (say $V^{\prime \prime}$ ) is involved, both kinds of ordered cumulants are identical, but in general a difference between them becomes apparent in third or higher order cumulants. The necessity of using $\overrightarrow{\mathbf{p}}$-cumulants to compute $M_{V}$ is explained in appendix $C$. There we also rederive the second 
equation for $C_{v}$, which reads

$$
\frac{\partial}{\partial t} C_{v}(t, t+\tau)=\left[M_{V}\left(t+\tau ; t / t_{0}\right)+N_{V}\left(t+\tau ; t / t_{0}\right)\right] C_{v}(t, t+\tau),
$$

where $M_{V}$ is the same as (3.2) and $N_{V}$ is given by

$$
N_{V}\left(t+\tau ; t / t_{0}\right)=\left\langle V^{\prime}(t): Q_{V^{\prime}}(t+\tau / t) Q_{\nu}\left(t / t_{0}\right):\right\rangle_{\overline{\mathrm{p}}}
$$

Note that compared to eq. (2.30c) of II not only p-cumulants have been changed into $\overline{\mathrm{p}}$-cumulants in (3.4) (for the same reason as in (3.2)), but also the matrix $V^{\prime}(t)$ has been shifted to the initial position in the cumulant. Again this affects only third and higher order cumulants.

To conclude we can summarize the situation by saying that all p-cumulants in sections 2, 3, 6, 7 and appendix $A$ of II should be replaced by $\bar{p}$-cumulants (the inhomogeneous case of section 4 of II is discussed in subsection 3.2 below).

\subsubsection{Discussion}

The replacement of p-cumulants by $\bar{p}$-cumulants does not lead to qualitatively different conclusions w.r.t. cluster property, long-time estimates etc. Therefore the estimates (3.1a) and (3.1b) of II are still valid. However the different position of $V^{\prime}(t)$ in (3.4) implies that the estimate (3.2b) of II is no longer valid (except in the commutative case, where $A(t)$ and $A\left(t^{\prime}\right)$ commute for all $t$ and $\left.t^{\prime}\right)$. It is still true that

$$
N_{V}\left(t+\tau ; t / t_{0}\right) \simeq N_{V}(t+\tau ; t /-\infty), \quad \text { if } t-t_{0} \gg \tau_{\mathrm{c}},
$$

but not that*

$$
N_{V}\left(t+\tau ; t / t_{0}\right) \simeq N_{V}\left(\infty ; t / t_{0}\right), \quad \text { if } \tau \gg \tau_{\mathrm{c}} .
$$

To see why this is the case consider a typical term of $N_{V}$ as in section 3 of II,

$$
\begin{aligned}
N_{V}^{(n, m)}= & \int_{t}^{t+\tau} \mathrm{d} s_{1} \ldots \int_{t}^{s_{n}-1} \mathrm{~d} s_{n} \int_{t_{0}}^{t} \mathrm{~d} t_{1} \\
& \ldots \int_{t_{0}}^{t_{m}-1} \mathrm{~d} t_{m}\left\langle V^{\prime}(t) V^{\prime \prime}\left(s_{1}\right) \ldots V^{\prime \prime}\left(s_{n}\right) \tilde{V}\left(t_{1}\right) \ldots \tilde{V}\left(t_{m}\right)\right\rangle_{\overline{\mathrm{p}}} .
\end{aligned}
$$

If $t-t_{1} \gg \tau_{\mathrm{c}}$, then because of the ordering of times, any matrix in the group $\left\{V^{\prime}(t), V^{\prime \prime}\left(s_{1}\right), \ldots, V^{\prime \prime}\left(s_{n}\right)\right\}$ is statistically independent of any matrix in the group $\left\{\tilde{V}\left(t_{1}\right), \ldots, \tilde{V}\left(t_{m}\right)\right\}$, so the $\overline{\mathrm{p}}$-cumulant vanishes. The same is true when $t_{1}-t_{2} \gg \tau_{\mathrm{c}}$,

\footnotetext{
$n=0$.

* Hence in the noncommutative case the second estimate of (3.5) of II can only be guaranteed for
} 
etc. Hence (3.5) follows. However if $s_{n}-t \gg \tau_{c}$ the cumulant does not necessarily vanish, although all matrices in the group $\left\{V^{\prime \prime}\left(s_{1}\right), \ldots, V^{\prime \prime}\left(s_{n}\right)\right\}$ are independent of those in the group $\left\{V^{\prime \prime}(t), \tilde{V}\left(t_{1}\right), \ldots, \tilde{V}\left(t_{m}\right)\right\}$ (compare (2.11)), except when the matrices $V$ commute at different times.

Conclusion. Secular terms as $t \rightarrow \infty$ are avoided by both equations (3.1) and (3.3), but secular terms as $\tau \rightarrow \infty$ only by (3.1) (except for the commutative case in which both expansions are valid). To get results which avoid both kinds of secular terms one should preferably solve (3.1) with initial condition $C_{v}(t, t)$ and calculate $C_{v}(t, t)$ itself by using the cumulant expansion for single-time averages (section 2 ).

Remark. The operators $L_{u}\left(t^{\prime}\right)$ in formula (7.7b) of II and $A^{(\kappa)}\left(t_{\kappa}\right)$ in (A.13) of II have to be shifted to the first position within the $\overline{\mathrm{p}}$-cumulant for the same reason as in $N_{V}$ above (eq. (3.4)).

\subsection{The inhomogeneous case}

The replacement of $\mathbf{p}$-cumulants by $\overline{\mathbf{p}}$-cumulants in the preceding subsection makes it necessary to modify the results for the inhomogeneous case in section 4.1 of II accordingly.

\subsubsection{Results}

The corrclation function $C_{v}(t, t+\tau)$ of the solution to the inhomogeneous equation (2.3) (fixed $u_{0}$ ) satisfies

$$
\frac{\partial}{\partial t} C_{v}(t, t+\tau)=G^{(1,1)} C_{v}(t, t+\tau)+G^{(2,1)}\langle v(t+\tau)\rangle+G^{(1,2)}\langle v(t)\rangle+G^{(2,2)},
$$

where

$$
G^{(1,1)}=M_{V}\left(t+\tau ; t / t_{0}\right)+N_{V}\left(t+\tau ; t / t_{0}\right)
$$

is the same as in (3.3). The quantities $G^{(2,1)}, G^{(1,2)}$ and $G^{(2,2)}$ can be obtained by the following prescription*. Expand $G^{(1,1)}$ in the joint moments or $t$-cumulants (but not just in $\overline{\mathrm{p}}$-cumulants) of $V^{\prime}$ and $V^{\prime \prime}$; to obtain $G^{(2,1)}$, replace the last matrix $V^{\prime}$ in each moment or $t$-cumulant contributing to $G^{(1,1)}$ by $g^{\prime}$; to obtain $G^{(1,2)}$ replace the last matrix $V^{\prime \prime}$ in each moment or $t$-cumulant by $g^{\prime \prime}$; and to obtain $G^{(2,2)}$, make both replacements. If a term in the expansion of $G^{(1,1)}$ contains no matrix $V^{\prime}$ (or $\left.V^{\prime \prime}\right)$, there is no corresponding contribution to $G^{(2,1)}$ and $G^{(2,2)}$ (or $G^{(1,2)}$ and $G^{(2,2)}$, respectively). Finally, the proviso has to be kept in mind that any matrix $C^{\prime}$ or

* Note that we do not define $\overline{\mathrm{p}}$-cumulants containing the vector $g$. 
$C^{\prime \prime}$ (where $C$ is a matrix $V$ or vector $g$ ) succeeding a matrix $g^{\prime}$ or $g^{\prime \prime}$ is replaced by $C$ (examples are given in the next subsection).

Results to second order in $V$ and/or $g$ are given by the formulas $(4.12 \mathrm{a}-\mathrm{d})$ of II which are still correct. The equation for $(\partial / \partial \tau) C_{v}(t, t+\tau)$ is the same as (3.7), but with $N_{V}$ in (3.8) omitted (and also the corresponding contributions to $G^{(2,1)}$, etc.).

\subsubsection{Discussion}

The prescription to obtain the contributions $G^{(2,1)} \ldots G^{(2,2)}$ from $G^{(1,1)}$, as given in the preceding subsection, can be derived by the same method as in II, section 4 (a more extensive rederivation has been given for the case of stochastic difference equations) $)^{4}$. The result we found there was essentially the same, except that the replacements $V^{\prime} \rightarrow g^{\prime}$ and $V^{\prime \prime} \rightarrow g^{\prime \prime}$ could be made in the p-cumulant itself, because no matrices are interchanged when the p-cumulant is expanded in moments or $t$-cumulants. But since we have in fact to use $\overline{\mathrm{p}}$-cumulants in $G^{(1,1)}$, one has to expand $\overline{\mathrm{p}}$-cumulants in $G^{(1,1)}$ first in moments or t-cumulants before making the replacements mentioned above*. For example, the following cumulant occurring in $G^{(1,1)}$ :

$$
\begin{aligned}
\left\langle V^{\prime \prime}(t+\tau) V^{\prime \prime}\left(t_{1}\right) V^{\prime}\left(t_{2}\right)\right\rangle_{\overline{\mathrm{p}}}= & \left\langle V^{\prime \prime}(t+\tau) V^{\prime \prime}\left(t_{1}\right) V^{\prime}\left(t_{2}\right)\right\rangle_{\mathrm{T}} \\
& -\left\langle V^{\prime \prime}(t+\tau) V^{\prime}\left(t_{2}\right)\right\rangle_{\uparrow}\left\langle V^{\prime \prime}\left(t_{1}\right)\right\rangle,
\end{aligned}
$$

yields the following contribution to $G^{(2,1)}$, etc.:

$$
\begin{aligned}
\text { to } G^{(2, t)}: & \left\langle V^{\prime \prime}(t+\tau) V^{\prime \prime}\left(t_{1}\right) g^{\prime}\left(t_{2}\right)\right\rangle_{\mathrm{t}}-\left\langle V^{\prime \prime}(t+\tau) g^{\prime}\left(t_{2}\right)\right\rangle_{\mathrm{t}}\left\langle V\left(t_{1}\right)\right\rangle, \\
\text { to } G^{(1,2):}: & \left\langle V^{\prime \prime}(t+\tau) g^{\prime \prime}\left(t_{1}\right) V\left(t_{2}\right)\right\rangle_{\mathrm{t}}-\left\langle V^{\prime \prime}(t+\tau) V^{\prime}\left(t_{2}\right)\right\rangle_{\mathrm{t}}\left\langle g^{\prime \prime}\left(t_{1}\right)\right\rangle, \\
\text { to } G^{(2,2):} & \left\langle V^{\prime \prime}(t+\tau) g^{\prime \prime}\left(t_{1}\right) g\left(t_{2}\right)\right\rangle_{\mathrm{t}}-\left\langle V^{\prime \prime}(t+\tau) g^{\prime}\left(t_{2}\right)\right\rangle_{\mathrm{t}}\left\langle g\left(t_{1}\right)\right\rangle .
\end{aligned}
$$

Here we made use of the fact that for any matrix $V$ and vectors $f$ and $g$,

$$
f \otimes V=V^{\prime \prime} f^{\prime}=f^{\prime} V ; \quad V \otimes f=V^{\prime} f^{\prime \prime}=f^{\prime \prime} V ; f \otimes g=f^{\prime} g=g^{\prime \prime} f .
$$

The contribution $N_{V}$ to $G^{(1,1)}$ and the corresponding contributions to $G^{(2,1)}$ etc. in general contain secular terms as $\tau \rightarrow \infty$ (the contributions to $G^{(2,2)}$ can be secular even in the commutative case, see subsection 3.3). In particular, to obtain the equilibrium correlation function $\lim _{t \rightarrow \infty} C_{v}(t, t+\tau)$ (assuming it exists) without secular terms in $\tau$, one cannot use the equation (3.7) with the 1.h.s. equal to zero and solve the resulting matrix equation, where the quantities $G^{(1,1)}, \ldots, G^{(2,2)}$ are expanded up to a certain order in $V$. That (3.7) could be used in the example of section 6 of II was because in that case we could evaluate $G^{(1,1)} \ldots G^{(2,2)}$ exactly. (next subscction). So in general the equation for $(\partial / \partial \tau) C_{v}$ has to be used $\left.{ }^{3}\right)$.

* The expression which then results can in general neither be written as a p-cumulant nor as a $\overline{\mathrm{p}}$-cumulant. 
To obtain the contribution $G^{(2,2)}$ corresponding to $M_{V}$ in (3.8) one has always to replace at least one matrix $V^{\prime}(s)$, originating from a matrix $\tilde{V}(s)$ in $Q_{\vec{D}}\left(t / t_{0}\right)$ (formula (3.2)), by a vector $g^{\prime}(s)$ with $s \leqslant t$. So all these contributions are transient if $\tau$ becomes large, i.e. $G^{(2,2)} \simeq 0$ if $\tau \gg \tau_{\mathrm{c}}$.

\subsubsection{Statistical independence}

If $V$ and $g$ in (2.3) are statistically independent the formula (4.13) of II (with $A$ replaced by $V$ and $f$ by $g$ ) is generally spoken again incorrect (see section 2). However in the special case that $A_{1}$ in (2.1) is delta-correlated as in (2.16) and statistically independent of $f$, the general result (3.7) reduces to the exact equation (the derivation is completely analogous to that in appendix B)

$$
\begin{aligned}
\frac{\partial}{\partial t} C_{v}(t, t+\tau)= & G^{(1,1)} C_{v}(t, t+\tau)+\left\langle g^{\prime}(t)\right\rangle\langle v(t+\tau)\rangle+\left\langle g^{\prime \prime}(t+\tau)\right\rangle\langle v(t)\rangle \\
& +\int_{t_{0}}^{t+\tau} \mathrm{d} s\left\langle\left\langle g^{\prime}(t)\left\{\left\langle Q_{V}(t+\tau / s)\right\rangle g(s)\right\}\right\rangle\right\rangle \\
& +\int_{t_{0}}^{t} \mathrm{~d} s\left\langle\left\langle g^{\prime \prime}(t+\tau)\left\{\left\langle Q_{V}(t+\tau / s)\right\rangle g(s)\right\}\right\rangle\right\rangle .
\end{aligned}
$$

Here $G^{(1,1)}$ is given by (3.8) and the brackets $\langle\langle\ldots\rangle\rangle$ denote an ordinary second order cumulant.

\section{Remarks:}

(i) In the example of the harmonic oscillator treated in section 6 of II, $K_{V}=0$ and $\left\langle Q_{V}(t+\tau / s)\right\rangle=1$ (compare subsection 2.2). Hence (3.9) indeed reduces to eq. (4.13) of II, which explains again why we got the correct results in section 6 of II.

(ii) The third and fourth lines of the r.h.s. of eq. (3.9) correspond to $N_{V}$ and $M_{V}$, respectively. If $\tau \gg \tau_{c}, g^{\prime \prime}(t+\tau)$ in the last line becomes independent of $g(s)$ (since $s \leqslant t$ ), so this term vanishes. But no such argument exists for the third line. In the limiting case that $g$ is also delta-correlated, the last line vanishes $(\tau>0)$, but the third line yields $\left\langle Q_{V}(t+\tau / t)\right\rangle$. This term in general produces secular terms in $\tau$ when expanded in powers of $V$. (In the harmonic oscillator problem this term turned out to be exactly equal to 1 , so no problems with secular terms arose.)

\section{Acknowledgements}

This investigation is part of the research program of the Stichting voor Fundamenteel Onderzoek der Materie (F.O.M.) which is financially supported by the Nederlandsc Organisatic voor Zuiver Wetenschappelijk Onderzoek (Z.W.O.). 


\section{Appendix A}

\section{Definition of the partially time ordered cumulants}

We give here the difinition of the p-cumulant (introduced in I) and the $\overline{\mathrm{p}}$-cumulant (introduced in section 3 of this paper).

Definition. To obtain a p- or $\overline{\mathrm{p}}$-cumulant of $m$ (noncommuting) matrices $A_{0}\left(t_{0}\right)$, $A_{1}\left(t_{1}\right), \ldots, A_{m-1}\left(t_{m-1}\right)$ where $t_{1} \geqslant \cdots \geqslant t_{m-1}$ use the following rules:

(i) Write a sequence of $m$ dots,

(ii) partition them into subsequences $\langle\ldots\rangle$ (denoting moments) by inserting angular brackets in all possible ways (excluding empty subsequences);

(iii) for each partition consisting of $p$ subsequences supply a factor $(-)^{p-1}$;

(iv) for each partition, write a zero on the first dot and any permutation of the numerals $1,2, \ldots, m-1$ on the remaining dots, subject to the condition that in each subsequence they must not decrease. Add all terms.

Finally use one of the following prescriptions:

( $p v$ ) Replace each numeral $n$ on the $k$ th $\operatorname{dot}$ by $A_{k-1}\left(t_{n}\right)$ : this yields $\left\langle A_{0}\left(t_{0}\right) \ldots A_{m-1}\left(t_{m-1}\right)\right\rangle_{\mathrm{p}}$

or

( $\bar{p} v)$ Replace each numeral $n$ by $A_{n}\left(t_{n}\right)$ : this yields $\left\langle A_{0}\left(t_{0}\right) \ldots A_{m-1}\left(t_{m-1}\right)\right\rangle_{\overline{\mathrm{p}}}$.

From this it follows that the two types of cumulants have the same time ordering, but a different matrix ordering. In a p-cumulant also vectors may occur (see I), as long as the expressions involved make sense as matrix products, but in a $\bar{p}$-cumulant this is in general impossible. Since the rules (i)-(iv) are the same for both cumulants, the decomposition in $t$-cumulants is the same as in appendix $A$ of $I$, with rule (A.v) replaced by (pv) or ( $\bar{p} v)$, respectively. In particular the cluster property still holds for both cumulants*. If all matrices $A_{i}, i=0,1, \ldots, m-1$ commute for all times, the $\overline{\mathrm{p}}$-cumulant reduces to the ordinary cumulant in the many variable case ${ }^{4}$ ), but the p-cumulant does not (in contrast to our assertion in subsection 2.1 of I). If all matrices are the same functions of time $A_{0}=\ldots=A_{m-1}$ ) the $\mathrm{p}$ - and $\overline{\mathrm{p}}$-cumulants are identical. Moreover, the difference becomes only apparent in third or higher order cumulants.

\section{Appendix B}

In this appendix we derive the result (2.17), (2.18) for the case that $A_{1}(t)$ in (2.1) has delta-correlated cumulants, i.e.

* Provided that all time-variables occur in decreasing order. 


$$
\begin{aligned}
& \left\langle A_{1}\left(t_{1}\right) \ldots A_{1}\left(t_{m}\right)\right\rangle_{\mathrm{p}}=D_{m}\left(t_{1}\right) \delta\left(t_{1}-t_{2}\right) \ldots \delta\left(t_{m-1}-t_{m}\right), \quad m \geqslant 2, \\
& \left\langle A_{1}\left(t_{1}\right)\right\rangle=D_{1}\left(t_{1}\right) .
\end{aligned}
$$

Consider again the equation (2.3) in the interaction representation

$$
\dot{v}(t)=V(t) v(t)+g(t), \quad v\left(t_{0}\right)=u_{0},
$$

where

$$
V(t)=\alpha \mathrm{e}^{-\left(t-t_{0}\right) A_{0}} A_{1}(t) \mathrm{e}^{\left(t-t_{0}\right) A_{0}}, \quad g(t)=\mathrm{e}^{-\left(t-t_{0}\right) A_{0}} f(t) .
$$

The formal solution of (B.2) is

$$
v(t)=Q_{V}\left(t / t_{0}\right) u_{0}+\int_{t_{0}}^{t} \mathrm{~d} s Q_{V}(t / s) g(s),
$$

where as before for any matrix $C(t)$

$$
Q_{C}\left(t / t_{0}\right)=\bar{T} \exp \int_{t_{0}}^{t} \mathrm{~d} s C(s)
$$

Average (B.3) and use the statistical independence of $V$ and $g$,

$$
\langle v(t)\rangle=\left\langle Q_{V}\left(t / t_{0}\right)\right\rangle u_{0}+\int_{t_{0}}^{t} \mathrm{~d} s\left\langle Q_{V}(t / s)\right\rangle\langle g(s)\rangle .
$$

Inserting (2.20) in (B.5) and differentiating it we find*

$$
\begin{aligned}
\langle\dot{v}(t)\rangle= & K_{V}\left(t / t_{0}\right)\langle v(t)\rangle+\langle g(t)\rangle \\
& +\int_{t_{0}}^{t} \mathrm{~d} s\left[\left\{K_{V}(t / s)-K_{V}\left(t / t_{0}\right)\right\} \overleftarrow{T} \exp \int_{s}^{t} \mathrm{~d} s^{\prime} K_{V}\left(s^{\prime} / s\right)\right]\langle g(s)\rangle .
\end{aligned}
$$

This equation is the general result for the case of statistically independent multiplicative and additive noise with fixed initial condition. The second and third term in the r.h.s. of (B.6) can also be found by expanding $F_{V}$ in (2.5b), using that $V$ and $g$ are independent.

Now a p-cumulant of $m V$-matrices has the form

$$
\begin{aligned}
\left\langle V(t) V\left(t_{1}\right) \ldots V\left(t_{m-1}\right)\right\rangle_{p}= & \alpha^{m} \mathrm{e}^{-\left(t-t_{0}\right) A_{0}} D_{m}(t) \mathrm{e}^{\left(t-t_{0}\right) A_{0}} \\
& \times \delta\left(t-t_{1}\right) \ldots \delta\left(t_{m-2}-t_{m-1}\right) .
\end{aligned}
$$

* This equation replaces eq. (1.2) of $I$. 
So

$$
\begin{aligned}
K_{V}\left(t / t^{\prime}\right) & \equiv\langle V(t)\rangle+\sum_{m=1}^{\infty} \int_{t_{0}}^{t} \mathrm{~d} t_{1} \ldots \int_{t_{0}}^{t_{m}}-\int^{-1} \mathrm{~d} t_{m}\left\langle V(t) V\left(t_{1}\right) \ldots V\left(t_{m}\right)\right\rangle_{\mathrm{p}} \\
& =\mathrm{e}^{-\left(t-t_{0}\right) A_{0}} \mathbb{D}(t) \mathrm{e}^{\left(t-t_{0}\right) A_{0}}
\end{aligned}
$$

where

$$
\mathbb{D}(t)=\sum_{m=1}^{\infty} \frac{\alpha^{m}}{m !} D_{m}(t) .
$$

Note that $K_{V}\left(t / t^{\prime}\right)$ is independent of $t^{\prime}$. So in (B.6) we have $K_{V}(t / s)=K_{V}\left(t / t_{0}\right)$ and in the original representation (B.6) results in the equation

$$
\langle\dot{u}(t)\rangle=K\left(t / t_{0}\right)\langle u(t)\rangle+\langle f(t)\rangle,
$$

where

$$
K\left(t / t_{0}\right)=A_{0}+\mathrm{e}^{\left(t-t_{0}\right) A_{0}} K_{V}\left(t / t_{0}\right) \mathrm{e}^{-\left(t-t_{0}\right) A_{0}}=A_{0}+\mathbb{D}(t) .
$$

This proves the validity of (2.17), (2.18) for the first $n$ components.

Next we calculate the second moment $\langle v(t) \otimes v(t)\rangle$ of the solution $v(t)$ of (B.2). From (B.3) one finds the following expression for the correlation function $C_{v}(t)=\langle v(t) \otimes v(t)\rangle$ :

$$
\begin{aligned}
C_{V}(t)= & \left\langle Q_{V^{(}}\left(t / t_{0}\right) Q_{V^{(}}\left(t / t_{0}\right)\right\rangle u_{0} \otimes u_{0} \\
& +\int_{t_{0}}^{t} \mathrm{~d} s\left\{\left\langle Q_{V^{(}}\left(t / t_{0}\right) Q_{V^{\prime}}(t / s)\right\rangle\left(u_{0} \otimes\langle g(s)\rangle\right)\right. \\
& \left.+\left\langle Q_{V^{\prime}}(t / s) Q_{V^{(}}\left(t / t_{0}\right)\right\rangle\left\langle\langle g(s)\rangle \otimes u_{0}\right)\right\} \\
& +\int_{t_{0}}^{t} \mathrm{~d} s \int_{t_{0}}^{t} \mathrm{~d} s^{\prime}\left\langle Q_{V^{\prime}}(t / s) Q_{V^{\prime}}\left(t / s^{\prime}\right)\right\rangle\left\langle g(s) \otimes g\left(s^{\prime}\right)\right\rangle,
\end{aligned}
$$

where we define for an arbitrary matrix or vector $C$,

$$
C^{\prime}=C \otimes \hat{\mathrm{l}}, \quad C^{\prime \prime}=\hat{\mathrm{i}} \otimes C, \quad \tilde{C}=C^{\prime}+C^{\prime \prime} .
$$

Here 1 denotes the unit matrix of the same dimension as $C$. Using the fact that $V^{\prime}(t)$ and $V^{\prime \prime}(t)$ commute for all $t$ and $t^{\prime}$, we can write

$$
Q_{V}\left(t / t^{\prime}\right) Q_{V^{\prime}}\left(t / t^{\prime}\right)=Q_{\vec{\nu}}\left(t / t^{\prime}\right) .
$$

Furthermore, the following factorization property holds, due to (B.1)

$$
\left\langle\Phi\left\{V(s), t_{1} \geqslant s \geqslant t_{2}\right\} \Psi\left\{V(s), t_{2} \geqslant s \geqslant t_{3}\right\}\right\rangle=\langle\Phi\rangle\langle\Psi\rangle,
$$


where $\Phi$ and $\Psi$ are arbitrary functionals of $V$ (or $V^{\prime}, V^{\prime \prime}, \tilde{V}$ ). For example, consider the average $\left\langle Q_{V}\left(t_{1} / t_{2}\right) Q_{V}\left(t_{2} / t_{3}\right)\right\rangle, t_{1} \geqslant t_{2} \geqslant t_{3}$. Defining the characteristic functional of $V$ for an arbitrary test function $k(t)$ with finite support by

$$
G_{V}[k]=\left\langle\overleftarrow{T} \exp \int_{-\infty}^{\infty} \mathrm{d} s k(s) V(s)\right\rangle,
$$

we find from (B.1) and the cumulant expansion (compare (B.7))

$$
G_{V}[k]=\overleftarrow{T} \exp \int_{-\infty}^{\infty} \mathrm{d} s L(s),
$$

where

$$
L(s)=\mathrm{e}^{-\left(s-t_{0}\right) A_{0}}\left\{\sum_{m=1}^{\infty} \frac{\alpha^{m}}{m !} k^{m}(s) D_{m}(s)\right\} \mathrm{e}^{\left(s-t_{0}\right) A_{0}} .
$$

So by choosing $k(s)=\theta\left(t_{1}-s\right) \theta\left(s-t_{3}\right)$, where $\theta(\cdot)$ denotes the Heaviside step function, we have

$$
\begin{aligned}
& \left\langle Q_{V}\left(t_{1} / t_{2}\right) Q_{V}\left(t_{2} / t_{3}\right)\right\rangle=\left\langle Q_{V}\left(t_{1} / t_{3}\right)\right\rangle=\bar{T} \exp \int_{t_{3}}^{t_{1}} \mathrm{~d} s L(s) \\
& =\left[\bar{T} \exp \int_{t_{2}}^{t_{1}} \mathrm{~d} s L(s)\right]\left[\bar{T} \exp \int_{t_{3}}^{t_{2}} \mathrm{~d} s L(s)\right]=\left\langle Q_{V}\left(t_{1} / t_{2}\right)\right\rangle\left\langle Q_{V}\left(t_{2} / t_{3}\right)\right\rangle .
\end{aligned}
$$

Applying (B.13) to (B.10) and using (B.12) one gets

$$
\begin{aligned}
C_{v}(t)= & \left\langle Q_{\vec{v}}\left(t / t_{0}\right)\right\rangle u_{0}^{\prime} u_{0}+\int_{t_{0}}^{t} \mathrm{~d} s\left\langle Q_{\vec{v}}(t / s)\right\rangle\langle\tilde{g}(s)\rangle\left\langle Q_{V}\left(s / t_{0}\right)\right\rangle u_{0} \\
& +\int_{t_{0}}^{t} \mathrm{~d} s \int_{t_{0}}^{s} \mathrm{~d} s^{\prime}\left\langle Q_{\tilde{v}}(t / s)\right\rangle\left\langle\tilde{g}(s)\left\langle Q_{V}\left(s / s^{\prime}\right)\right\rangle g\left(s^{\prime}\right)\right\rangle .
\end{aligned}
$$

Here we made use of the fact that for any matrix $V$ and vectors $f$ and $g$,

$$
f \otimes V=V^{\prime \prime} f^{\prime}=f^{\prime} V ; \quad V \otimes f=V^{\prime} f^{\prime \prime}=f^{\prime \prime} V ; f \otimes g=f^{\prime} g=g^{\prime \prime} f .
$$

Using (2.20) we find from (B.14)

$$
\begin{aligned}
\dot{C}_{v}(t)= & K_{\tilde{V}}\left(t / t_{0}\right) C_{v}(t)+\langle\tilde{g}(t)\rangle\left\langle Q_{V}\left(t / t_{0}\right)\right\rangle u_{0} \\
& +\int_{t_{0}}^{t} \mathrm{~d} s\left\langle g(t)\left\langle Q_{v}(t / s)\right\rangle g(s)\right\rangle,
\end{aligned}
$$


since again $K_{\vec{v}}\left(t / t_{0}\right)=K_{\mathcal{V}}(t / s)$ due to (B.1). If (B.5) is inserted in (B.15) there results

$$
\dot{C}_{v}(t)=K_{\tilde{v}}\left(t / t_{0}\right) C_{v}(t)+\langle\tilde{g}(t)\rangle\langle v(t)\rangle+\int_{t_{0}}^{t} \mathrm{~d} s\left\langle\left\langle\tilde{g}(t)\left\{\left\langle Q_{V}(t / s)\right\rangle g(s)\right\}\right\rangle\right\rangle .
$$

If we transform back to the original representation we finally arrive at the results (2.15), (2.17) and (2.18) for $\langle u(t) \otimes u(t)\rangle$.

\section{Appendix C}

In this appendix we rederive the equations (3.1)-(3.4). In II, section 2.2, the following expression for the correlation function $C_{v}(t, t+\tau)=\langle v(t) \otimes v(t+\tau)\rangle$ of the solution $v(t)$ to $(2.3)$ in the homogeneous case $(g(t)=0)$ was found

$$
C_{v}(t, t+\tau)=\left[\bar{T} \exp \int_{t_{0}}^{\infty} \mathrm{d} s K_{B}\left(s / t_{0}\right)\right] u_{0} \otimes u_{0}
$$

$K_{B}$ is defined as in (2.5a) and

$$
B(s)=\left\{\begin{aligned}
\tilde{V}(s), & t_{0} \leqslant s \leqslant t, \\
V^{\prime \prime}(s), & t<s \leqslant t+\tau, \\
0, & \text { otherwise, }
\end{aligned}\right.
$$

where $\tilde{V}$ and $V^{\prime \prime}$ are defined by (2.14). We then proceeded to express $K_{B}\left(s / t_{0}\right)$ in terms of the p-cumulants of $V^{\prime}$ and $V^{\prime \prime}$. However, the definition given in I (and appendix A of this paper) of the p-cumulant is not quite applicable here. This can easily be demonstrated by considering the third order cumulant $\left\langle B\left(t_{1}\right) B\left(t_{2}\right) B\left(t_{3}\right)\right\rangle_{\mathrm{p}}$, with $t_{1}>t_{2}>t_{3}$. Using the decomposition in t-cumulants (appendix $\mathrm{A}$ of $\mathrm{I}$ ) we find

$$
\left\langle B\left(t_{1}\right) B\left(t_{2}\right) B\left(t_{3}\right)\right\rangle_{\mathrm{p}}=\left\langle B\left(t_{1}\right) B\left(t_{2}\right) B\left(t_{3}\right)\right\rangle_{\mathrm{t}}-\left\langle B\left(t_{1}\right) B\left(t_{3}\right)\right\rangle_{\mathrm{t}}\left\langle B\left(t_{2}\right)\right\rangle .
$$

Since each t-cumulant is a sum of terms which all have the same order of the time variables occurring in them, we use (C.2) to write (C.3) as

$$
\left\langle B\left(t_{1}\right) B\left(t_{2}\right) B\left(t_{3}\right)\right\rangle_{\mathrm{p}}=\left\langle V^{\prime \prime}\left(t_{1}\right) V^{\prime \prime}\left(t_{2}\right) \tilde{V}\left(t_{3}\right)\right\rangle_{\mathrm{t}}-\left\langle V^{\prime \prime}\left(t_{1}\right) \tilde{V}\left(t_{3}\right)\right\rangle_{\mathrm{t}}\left\langle V^{\prime \prime}\left(t_{2}\right)\right\rangle .
$$

Now (C.4) cannot be written as $\left\langle V^{\prime \prime}\left(t_{1}\right) V^{\prime \prime}\left(t_{2}\right) \tilde{V}\left(t_{3}\right)\right\rangle_{\mathrm{p}}$, since in the second term on the r.h.s. of (C.4) not only the time variables $t_{2}$ and $t_{3}$ are interchanged (compared to the first term), but also the matrices $V^{\prime \prime}$ and $\tilde{V}$ themselves, contrary to the definition of the p-cumulant. Therefore, we introduce the $\overline{\mathrm{p}}$-cumulant as defined in appendix $\mathrm{A}$, so that (C.4) can be written as $\left\langle V^{\prime \prime}\left(t_{1}\right) V^{\prime \prime}\left(t_{2}\right) \tilde{V}\left(t_{3}\right)\right\rangle_{\bar{p}}$. 
By similar arguments one can convince onself that all p-cumulants of matrices $B$ occurring in (C.1) can be written as $\overline{\mathrm{p}}$-cumulants of $V^{\prime \prime}$ and $\tilde{V}$. Therefore, we conclude that

$$
K_{B}\left(s / t_{0}\right)=\left\{\begin{aligned}
K_{\mathcal{D}}\left(s / t_{0}\right), & t_{0} \leqslant s \leqslant t, \\
M_{\nu}\left(s ; t / t_{0}\right), & t<s \leqslant t+\tau, \\
0, & \text { otherwise. }
\end{aligned}\right.
$$

Here $K_{\tilde{V}}$ is defined in (2.3b) (since only matrices $\tilde{V}$ are involved one may use por $\overline{\mathrm{p}}$-cumulants), whereas $M_{V}$ is defined by

$$
M_{V}\left(s ; t / t_{0}\right)=\left\langle V^{\prime \prime}(s): Q_{V^{m}}(s / t) Q_{\mathfrak{V}}\left(t / t_{0}\right):\right\rangle_{\overline{\mathrm{p}}} .
$$

From (C.1) and (C.5) eq. (3.1) immediately follows.

We now give also a new derivation of the equation for $(\partial / \partial t) C_{v}(t, t+\tau)$, since that in II, scction 2.3 , is strictly spoken only correct in the commutative case $\left(\left[V(t), V\left(t^{\prime}\right)\right]=0\right.$ for all $t$ and $\left.t^{\prime}\right)$. The reason is that the $\bar{T}$ operator has been defined so as to order the matrices $M_{V}\left(s ; t / t_{0}\right)$ for $s>t$, but this does not necessarily imply that it also orders the matrices $N_{V}\left(s ; t / t_{0}\right)$ as defined in II. However the argument can be corrected as follows. For $C_{v}$ we have the following expression by direct integration of (2.3):

$$
C_{v}(t, t+\tau)=\left\langle Q_{V^{\prime}}(t+\tau / t) Q_{\vec{v}}\left(t / t_{0}\right)\right\rangle u_{0} \otimes u_{0} .
$$

Differentiating (C.7) w.r.t. the variable $\tau$ and eliminating $u_{0} \otimes u_{0}$ from the result by means of (C.7) one finds

$$
\frac{\partial}{\partial \tau} C_{v}(t, t+\tau)=\mathscr{L} C_{v}(t, t+\tau)
$$

where

$$
\mathscr{L}=\left\langle V^{\prime \prime}(t+\tau) Q_{V^{\prime \prime}}(t+\tau / t) Q_{\mathcal{P}}\left(t / t_{0}\right)\right\rangle\left\langle Q_{V^{\prime \prime}}(t+\tau / t) Q_{\vec{D}}\left(t / t_{0}\right)\right\rangle^{-1},
$$

which obviously has to be identified with $M_{V}\left(t+t ; t / t_{0}\right)$ in (3.2). This can be checked by expanding all $Q$-matrices in (C.8) in powers of $V^{\prime \prime}$ or $\tilde{V}$, collecting all terms with a certain number of matrices $V^{\prime \prime}$ or $\tilde{V}$ and writing the result as an ordered integration,

$$
\int_{t}^{t+\tau} \mathrm{d} s_{1} \ldots \int_{t}^{s_{m}-2} \mathrm{~d} s_{m-1} \int_{t_{0}}^{t} \mathrm{~d} t_{m} \int_{t_{0}}^{t_{m}} \mathrm{~d} t_{m+1} \ldots \int_{t_{0}}^{t_{n}-1} \mathrm{~d} t_{n}[\ldots],
$$

which then agrees with the expression obtained by expanding the $\overrightarrow{\mathrm{p}}$-cumulant (3.2) to the same order. 
Differentiation of (C.7) w.r.t. the variable $t$ yields

$$
\frac{\partial}{\partial t} C_{v}(t, t+\tau)=\left(\mathscr{L}+\mathscr{L}^{\prime}\right) C_{v}(t, t+\tau),
$$

where $\mathscr{L}$ is the same as (C.8) and $\mathscr{L}^{\prime}$ is given by

$$
\mathscr{L}^{\prime}=\left\langle V^{\prime}(t) Q_{V^{\prime}}(t+\tau / t) Q_{\mathfrak{v}}\left(t / t_{0}\right)\right\rangle\left\langle Q_{V^{\prime}}(t+\tau / t) Q_{\mathfrak{v}}\left(t / t_{0}\right)\right\rangle^{-1} .
$$

Here we have used that $V^{\prime}$ and $V^{\prime \prime}$ commute to shift $V^{\prime}(t)$ to the first position in the first factor of $\mathscr{L}^{\prime}$. Now we can apply the same procedure as above (expanding in powers of $V^{\prime \prime}$ and $\tilde{V}$, writing the results as an ordered integration) to find the $(n)$ th order contribution to (C.9). The only difference with $\mathscr{L}$ is that each term of the $n$th order contribution contains a matrix $V^{\prime}(t)$ instead of $V^{\prime \prime}(t+\tau)$ on the first position of the product of joint moments of which each term consists. Hence (C.9) can be identified with (3.2) where $V^{\prime \prime}(t+\tau)$ is replaced by $V^{\prime}(t)$. This yields (3.4).

\section{References}

1) J.B.T.M. Roerdink, Physica 109A (1981) 23.

2) J.B.T.M. Roerdink, Physica 112A (1982) 557.

3) An explicit calculation can be found in: J.B.T.M. Roerdink, thesis, Utrecht, 1983, chap. III, section 6.1.

4) J.B.T.M. Roerdink, Physica 120A (1983) 153.

5) E. Meeron, J. Chem. Phys. 27 (1957) 1238, appendix. 